\title{
Log Likelihood Ratio Based Relay Selection Scheme for Amplify and Forward Relaying with Three State Markov Channel
}

\author{
Manish Sahajwani ${ }^{1, * \mathbb{D}}$, Alok Jain ${ }^{2}$ and Radheyshyam Gamad ${ }^{3}$ \\ 1 Department of Electrical and Electronics, IES IPS Academy, 452012 Indore (M.P.), India \\ 2 Department of Electronics and Instrumentation in SATI, 464001 Vidisha (M.P.), India; \\ alokjain6@rediffmail.com \\ 3 Department of Electronics and Instrumentation SGSITS, 452003 Indore (M.P.), India; rsgamad@gmail.com \\ * Correspondence: msahajwanister@gmail.com
}

Received: 21 June 2018; Accepted: 3 September 2018; Published: 6 September 2018

\begin{abstract}
This paper presents log likelihood ratio (LLR) based relay selection scheme for a cooperative amplify and forward relaying system. To evaluate the performance of the aforementioned system model, a three state Markov chain based fading environment has been presented to toggle among Rayleigh, Rician, and Nakagami-m fading environment. A simulation is carried out while assuming that there is no possibility of direct transmission from the source and destination terminal. Simulation results on the basis of Bit Error Rate (BER), Instantaneous Channel Capacity, and Outage probability have been presented and compared for different cases. In each case, the best performance of the proposed algorithm is obtained with a Binary Phase Shift Keying (BPSK) modulation scheme.
\end{abstract}

Keywords: cooperative communication; optimal relay selection; finite state markov channel; multiple input multiple output (mimo); cooperative diversity; log-likelihood ratio

\section{Introduction}

The relentless demand for data in wireless networks has revealed some communication constraints. In fact, wireless links have always had a lower bandwidth limit than wired links. This limit forces users to use applications reminiscent of wired networks from several decades. New technologies like multiple-input and multiple-output (MIMO) systems increase the bandwidth in number of bits per second per hertz with the help of techniques like spatial multiplexing, and improve the reliability (and coverage) of the wireless link by exploiting the spatio-temporal coding and the directionality of the antennas (beamforming). However, all these improvements can be realized at the cost of several radio frequency $(\mathrm{RF})$ components at the transmitter and the receiver. In addition, the size of mobile equipment may limit the number of antennas to be deployed. Even if MIMO technology is feasible, other constraints on the allocation of frequency bands to users are encountered due to historical and regulatory reasons. With this limitation, the free frequency bands show a lot of interference because of the large number of users. In order to solve these problems, the concept of cooperation between users is introduced. In a cooperative communication, several relay nodes operate together with the source node to transmit the information to the destination. Therefore, the exploitation of MIMO techniques becomes possible without the need to locate several antennas at the nodes of the network. Communication thus benefits from spatial diversity since the signals are transmitted over independent fading channels, which ensures better detection to the receiver. Cooperation techniques take advantage of the broadcasting property of the wireless signals. The fact that the signal from a source to a destination can be "listened to" by neighboring nodes allows the later to process the "listen" signal and to transmit it to the destination. The concept of cooperative diversity emerged with the 
study of a multi-way channel proposed by Van Der Meulen, where the author discussed the problem associated with the three terminal communication system and provided bounds on the capacity of the relay channels [1,2]. Sendonaris et al. were first to propose the application of diversity to enhance system performance, the authors have proposed a cooperative beamforming where source and relay nodes perform a phase adjustment to facilitate the coherent reception of multiple copies at destination node [3,4]. A major work on a cooperative diversity scheme was done by Laneman et al., namely, fixed relaying, incremental relaying, and selected relaying with single source-relay and destination with no availability of channel state information (CSI) at transmitter end $[5,6]$. To overcome the problem of spectrum inefficiency caused by use of relaying terminals, relay-selection schemes have been proposed in the literature. Several researchers have proved the significance of using log-likelihood ratio over SNR based schemes as log likelihood ratio (LLR) magnitude provides the reliability of hard decision. Therefore, this paper presents a LLR based dual-relay selection scheme for cooperative amplify and forward relaying system. Furthermore, a time varying characteristic of the fading environment is modelled through a three state Markov Channel.

The rest of this paper is organized as follows: In Section 2, related work on the relay selection in a cooperative communication system has been elaborated. In Section 3, a LLR based dual relay selection scheme is discussed. In Section 4, the simulation results for the proposed scheme have been presented and, finally, Section 5 concludes this paper.

\section{Related Work}

Some important works on relay selection include Bletsas et al. $[7,8]$, where the authors present a simple approach to select the best possible candidate to support the source node in forwarding its information with the help of the measurement of the instantaneous channel conditions. Siriwongpairat et al. [9] have proposed a threshold selection based relaying scheme for a cooperative decode and forward system. Michalopoulos [10] has presented an optimized user selection approach for amplifying and forward relaying method. The performance of cooperative diversity systems can be further enhanced through proper resource selection. In this regards, B. Kumbhani and R.S. Kshetrimayum have derived a closed form of expression for the outage probability for a transmit antenna selection MIMO system under a $K-\mu$ fading channel [11]. Practical application of the cooperative relaying technique in a cellular network is discussed by A. Papadogiannis and G. $\mathrm{C}$ Alexandropoulos. Authors have evaluated the performance of proactive and reactive relay selection in cooperative relaying techniques over a dynamic Nakagami-m fading channel in the presence of inter channel interference for a cellular network for an adaptive transmission scenario and calculated the simulation results based on the average system capacity and outage probability for various cases of fading [12]. In another work, G. C Alexandropoulos et al. [13] presented a generalized framework for a DF relaying scheme and derived a closed-form expression for outage probability and symbol error rate for repetitive transmission technique and relay selection technique for different modulation schemes under a Nakagami-m fading channel. The authors have shown that relay selection based techniques are useful only when the channel state information for relay node is perfect. L. Xuet al. has analyzed the performance of a decode and forward relaying scheme for transmit antenna selection. The authors have also analyzed the outage probability of a system for a Nakagami fading environment [14]. To improve the performance of a multi-user cooperative AF system, a user-relay assignment based antenna selection scheme is presented by $Z$. Bai et al. [15]. The proposed scheme uses best relay selection in conjunction with an antenna selection to improve the throughput performance of the system. The application of a cooperative relay selection to overcome the problem of data transmission in a power line communication (PLC) system is presented by B. Nikfar and A. J. H. Vinck [16]. The authors have proposed a two-hop communication scenario to overcome the transmission losses in a PLC system. In other work, the application of a relay selection scheme is used to improve the secrecy outage probability in half-duplex cognitive radio networks [17]. The authors have proposed a K-best relay selection scheme for optimal relay selection and partial relay selection and derived a 
closed form of expression for secrecy outage probability in the case of multiple eavesdroppers and multiple primary users. Alexandropoulos et al. have analyzed the performance of dual hop-DF relaying scheme with pure and rate-selective relay selection over a Nakagami fading environment. It is assumed that the direct link between source-to-destination is active and relay selection is done only when the direct link is weak. The authors have also derived closed-form expression for outage probability and average symbol error probability (ASEP) using MGF (moment gathering function) and CDF (cumulative distribution function) [18]. In another seminal work, Alexandropoulos analyzed the performance of a dual-hop DF relaying scheme over an arbitrary Nakagami fading channel and derived the closed-form expression for (ASEP) using MGF [19]. USH verma et al. [20] presented the application of a cooperative relay selection scheme to improve the data rate and throughput performance of a LTE system. The proposed relay selection scheme uses error rate performance as a selection criterion. CMK swain and S Das [21] have investigated the performance of a cooperative relay selection on an IEEE 802.16j MMR WiMAX network. The authors used a MAX-MIN and Harmonic Mean relay selection criterion to analyze the system performance. Recent research has shown the significance of the $\log$ likelihood ratio in improving the performance of a communication system. Several researchers have also mentioned its importance in improving the performance of cooperative diversity systems. S.W. Kim and E.Y. Kim [22] proposed an optimal antenna selection scheme to minimize bit error probability based on a log-likelihood ratio test. Palat et al. [23] have suggested an LLR based adaptive relaying technique based on a selective decode and forward transmission strategy and provided expression for optimal LLR threshold for minimum average bit error rate (ABER) requirement. A. Mahdy and A. Walled [24] have provided a LLR threshold based relay selection and channel estimation scheme for cooperative decode and forward relaying technique using Binary Phase Shift Keying (BPSK) modulation. Most of the research work on the cooperative diversity approach does not contain the time-varying characteristics of fading channels. Since wireless propagation environment changes its nature depending on various things, it is always a good option to characterize a fading channel as a Markov chain with the limited number of stages based on an actual variation of the channel. H.S.Wang and N. Moayeri [25] were first to provide the application of the Markovian process in fading channels and have given the idea of a finite-state Markov channel (FSMC). Later Q. Zhang and S.A. Kassam [26] formulated the finite state Markov channel for Rayleigh fading environment through partitioning a received SNR in different states. Babich et al. [27] provided a generalized Markov model to represent flat fading environment with the impact of white Gaussian noise.

\section{System Model}

Let us consider a network comprised of three types of devices: a source, $n$-relays and a destination, all equipped with a single antenna. The relay nodes do not provide any additional traffic to the destination and the nodes are assumed to be half-duplex, that is, one node cannot transmit and receive simultaneously. The transmission of information occurs during two temporal phases of the same duration. The first phase is known as the "broadcast phase", where the source node broadcasts its information without redundancy during symbol periods. The relay nodes amplify (or detect and decode then re-encode) the symbols received and transmits it in the "relaying phase" where the relay nodes send the received symbols (refer Figure 1). It is assumed that the source-destination link is corrupt, so the destination does not take into account the signal originally transmitted in the broadcast phase.

According to the given system model, the received signal at $N^{\text {th }}$ relay in first phase can be given as:

$$
S R_{N}=\sqrt{E_{S}} S R_{N} * \chi+\eta_{S R N}
$$

where $H_{S R_{N}}$ is the channel coefficient present between source and $N^{\text {th }}$ relay, $\chi$ represent transmitted symbol from source, $\eta_{S R N} \sim C N\left(0, N_{0}\right)$ represents the additive white Gaussian noise (AWGN) noise with zero mean and $N_{0}$ variance and $\sqrt{E_{s}}$ presents the average energy per symbol. In the next step, 
the best relay to destination pair is selected using a Log Likelihood ratio to forward the received signal in its first phase. The best relay pair $b_{i}$ is selected such that:

$$
b_{i}=\underset{i \in N}{\operatorname{argmax}}\left\{L L R_{N}\right\} \forall i \in(1,2)
$$

where $L L R_{N}$ represents $L L R$ magnitude of signal received at $N^{\text {th }}$ relay in first phase given as:

$$
L L R_{N}=\frac{4 \sqrt{E_{s}}}{N_{0}} \operatorname{Re}\left\{H_{S R_{N}}^{*} Z_{S R_{N}}\right\}=\left|H_{S R_{N}}\right|^{2} \cdot x+\operatorname{Re}\left\{H_{S R_{N}}^{*} \eta_{S R N}\right\}
$$

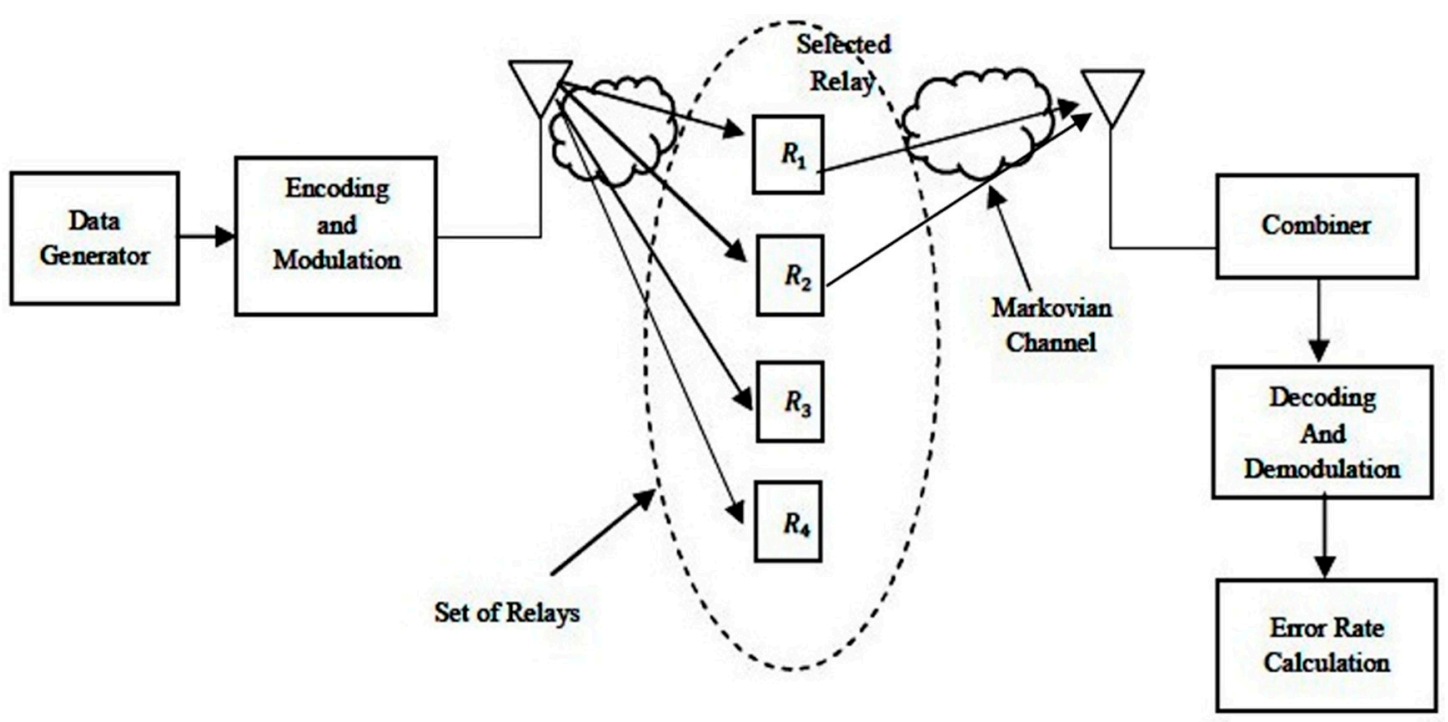

Figure 1. General block diagram of cooperative diversity system.

The magnitude of $L L R$ in Equation (3) represents the reliability of hard decision whereas its sign represents hard decision value. Furthermore, the bit error probability at $i$ th relay i.e., $P_{b i}$ is related to LLR magnitude as [22]:

$$
P_{b i}=\frac{1}{1+e^{\left|L L R_{N}\right|}}
$$

According to the relationship provided in Equation (4), relay with maximum $L L R$ magnitude will result in the lowest error possible. In the second phase, the selected relay pair will forward the signal received in the first phase with an amplification factor given as $\beta_{i}=\frac{1}{\sqrt{\sqrt{E_{s}}\left|H_{s r_{i}}\right|^{2}+N_{0}}} i \in(1,2)$. Therefore, the received signal at the destination after the second phase of the transmission is given as:

$$
\mathrm{Z}_{R_{i} D}=\sqrt{E_{S}} H_{R_{i} D} * \beta_{i}\left(\sqrt{E_{S}} H_{S R_{N}} * \chi+\eta_{S R N}\right)+\eta_{R_{i} D} \forall i \in(1,2)
$$

At the receiver end, the signal received from the two relays are combined using a maximal ratio combining (MRC), the combined signal will be:

$$
\tilde{\chi}=\delta_{1} H_{R_{1} D}^{*} Z_{R_{1} D}+\delta_{2} H_{R_{2} D}^{*} Z_{R_{2} D}
$$

where $Z_{R_{1} D}, Z_{R_{2} D}$ represents signal received at from relay- 1 and relay- 2 respectively and parameters $\delta_{1}$ and $\delta_{2}$ are known as link weights, the values of these link weights are determined such that signal strength of received signal at MRC output is maximum. These weights can be calculated as $\delta_{1}=\frac{\sqrt{E_{s}} H_{R_{1}}^{*} D}{N_{0}}$ and $\delta_{2}=\frac{\sqrt{E_{s}} H_{R_{2} D}^{*}}{N_{0}}$. Assuming the average energy of transmitted signal $\chi$ to be unity, the value of instantaneous SNR $\left(\gamma_{t}\right)$ at the destination node using the MRC receiver can be given as: 


$$
\gamma_{t}=\sqrt{E_{s}}\left(\frac{\left|H_{R_{1} D}\right|^{2}+\left|H_{R_{2} D}\right|^{2}}{N_{0}}\right)
$$

Considering the case of a coherent BPSK Modulation scheme, the conditional Bit Error Rate Probability for L-Branch diversity, $P_{b}\left(E \mid\left\{\gamma_{l}\right\}_{l=1}^{L}\right)$ can be given as [28]:

$$
P_{b}\left(E \mid\left\{\gamma_{l}\right\}_{l=1}^{L}\right)=Q\left(\sqrt{2 g \gamma_{t}}\right)
$$

For coherent BPSK modulation, $g=1$, and $Q($.$) represents Gaussian Q$ function.

The average Bit Error Rate of binary signals using the probability density function (PDF) of the corresponding fading distribution can be given as:

$$
P_{b}=\int_{0}^{\infty} Q\left(\sqrt{2 g \gamma_{t}}\right) p_{\gamma_{t}}\left(\gamma_{t}\right) d \gamma_{t}
$$

where $p_{\gamma_{t}}$ represents fading distribution of $\gamma_{t}$. According to the considered case of the three state Markov channel, the $p_{\gamma_{t}}$ can toggle among Rayleigh, Rician and Nakagami distribution and its corresponding value will be:

Case-1: For Rayleigh Fading Environment

$$
p_{\gamma_{l}}\left(\gamma_{l}\right)=\frac{1}{\bar{\gamma}} e^{-\gamma_{l} / \bar{\gamma}}
$$

where $\gamma_{l}$ represents SNR per bit per path and $\bar{\gamma}$ represents SNR per bit.

Case-2: For Rician Fading Environment

$$
p_{\gamma_{l}}\left(\gamma_{l}\right)=\frac{2(K+1) \gamma_{l}}{\Omega} e^{\left(-K-\frac{(K+1) \gamma_{l}^{2}}{\Omega}\right) I_{0}\left(2 \sqrt{\frac{K(K+1)}{\Omega}} \gamma_{l}\right)}
$$

where $K$ represents ratio between the power in direct path and indirect path, and $\Omega$ represents total available power from all paths. Furthermore, $I_{0}($.$) represents modified Bessel function of 0$ th order.

Case-3: For Nakagami Fading Environment

$$
p_{\gamma_{l}}\left(\gamma_{l}, m, \omega\right)=\frac{2 m^{m} \gamma_{l}^{2 m-1}}{\Gamma(m) \omega^{m}} e^{\left(-\frac{m \gamma_{l}^{2}}{\omega}\right)} \text { for } m \geq \frac{1}{2}, \text { and } \omega>0
$$

where $m$ is known as shaping parameter and $\omega$ is controlling spread.

This section is further explained to provide a concise and precise description of the experimental results, their interpretations and the experimental conclusions that can be drawn.

\section{Finite State Markov Channel Modeling}

The channel coefficients between source-to-relay and relay-to-destination links are assumed to be modeled with the Markovian process with three states representing a transition between Rayleigh, Rician, and Nakagami fading environments as shown in Figure 2. 


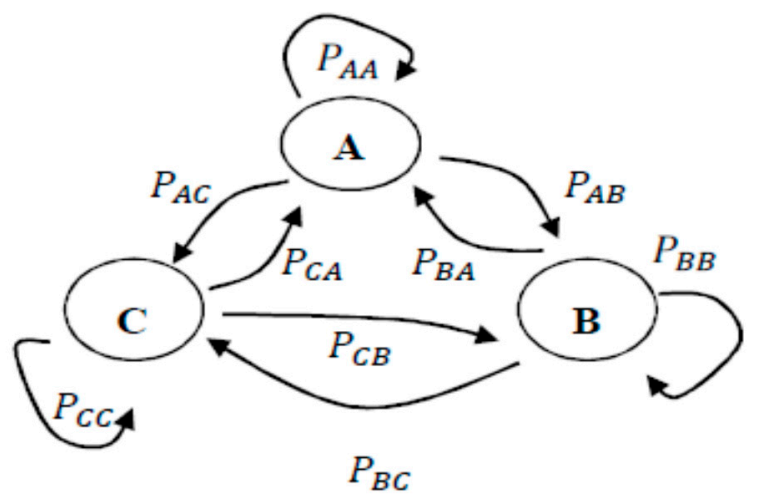

Figure 2. Three-State Markov process with state-A being Rayleigh Fading, State-B represents Rician Fading and State-C represents Nakagami-m fading channel.

State-A represents Rayleigh fading environment, state-B represents Rician fading environment and State-C represents Nakagami fading environment with $P_{A B}$ being transition probability from Rayleigh to Rician fading, $P_{B C}$ being transition probability from Rician to Nakagami fading and $P_{C A}$ represents transition probability with Nakagami to Rayleigh fading environment and vice versa. These transition probabilities can be given by transition probability matrix P:

$$
P=\left[\begin{array}{lll}
P_{A A} & P_{A B} & P_{A C} \\
P_{B A} & P_{B B} & P_{B C} \\
P_{C A} & P_{C B} & P_{C C}
\end{array}\right]
$$

and corresponding steady state vector for three state Markov chain is given by:

$$
\Pi_{3 s t a t e s}=\left[\pi_{1} \pi_{2} \pi_{3}\right]
$$

where $\pi$ is the steady state probability vector and $\pi_{1}, \pi_{2}, \pi_{3}$ are steady state probabilities for state A, state $B$, and state $C$ respectively.

Based on the values defined in Equations (13) and (14) value of $p_{\gamma_{l}}$ can be determined, which follow Rayleigh, Rician, or Nakagami fading environment.

\section{Results and Discussion}

This paper presents a LLR based relay selection scheme over three state Markov channel model. MATLAB based simulation has been presented, consisting of a single antenna source, destination, and a set of relaying nodes. The simulation is carried out by considering a three-state Markovian process where the first state represents a Rayleigh fading environment, the second state represents a Rician fading environment, and the third state represents a Nakagami fading environment. To simulate system performance, four cases of transition probability have been considered. For the first case, transition probability to toggle among Rayleigh, Rician, and Nakagami fading is set to be equal; i.e., 1/3-and accordingly a transition probability matrix has been defined. The second case represents the simulation of a Rayleigh fading channel through Markov chain, transition probability moving from any state to a state representing Rayleigh fading channel is set to 1, as provided in the transition probability matrix defined for the Rayleigh fading scenario. Similarly, the transition probability matrix for a Rician and Nakagami fading scenario using a Markov chain has been created and defined through their transition probability matrix, respectively. Simulation parameters as discussed above are presented in Table 1. Furthermore, a general case of transition probability representing random variation among Rayleigh, Rician, and Nakagami fadings is also presented in Table 1 for reference purposes. Bit Error Rate (BER) and Channel Capacity are used to evaluate the system performance. 
Table 1. Simulation Parameters.

\begin{tabular}{|c|c|c|c|c|c|}
\hline Modulation Scheme & \multicolumn{5}{|c|}{ BPSK, QPSK } \\
\hline Power Allocation Factor & \multicolumn{5}{|c|}{0.5} \\
\hline SNR Range & \multicolumn{5}{|c|}{$0: 2: 20$} \\
\hline Transition Probability $P$ (General case for Example) & \multicolumn{5}{|c|}{ 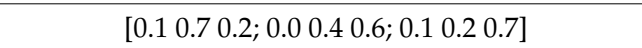 } \\
\hline Fading environment & \multicolumn{5}{|c|}{$\begin{array}{c}\text { Three state Markov channel with Rayleigh, Rician, } \\
\text { and Nakagami channels }\end{array}$} \\
\hline Frame Length & \multicolumn{5}{|c|}{256 bit } \\
\hline Variance S-R, R-D and S-D link & \multicolumn{5}{|c|}{1} \\
\hline Transition probability matrix with equal probability & $P=[$ & $\begin{array}{l}1 / 3 \\
1 / 3 \\
1 / 3 \\
\end{array}$ & $\begin{array}{l}1 / 3 \\
1 / 3 \\
1 / 3\end{array}$ & $\begin{array}{l}1 / 3 \\
1 / 3 \\
1 / 3\end{array}$ & \\
\hline Transition probability matrix with Rayleigh only & $P=$ & $\begin{array}{l}1 \\
1 \\
1\end{array}$ & $\begin{array}{l}0 \\
0 \\
0\end{array}$ & $\begin{array}{l}0 \\
0 \\
0\end{array}$ & \\
\hline Transition probability matrix with Rician only & $P=$ & $\begin{array}{l}0 \\
0 \\
0\end{array}$ & $\begin{array}{l}1 \\
1 \\
1\end{array}$ & $\begin{array}{l}0 \\
0 \\
0\end{array}$ & \\
\hline Transition probability matrix with Nakagami only & $P=$ & $\begin{array}{l}0 \\
0 \\
0\end{array}$ & $\begin{array}{l}0 \\
0 \\
0\end{array}$ & $\begin{array}{l}1 \\
1 \\
1\end{array}$ & \\
\hline
\end{tabular}

Bit Error rate comparison for proposed LLR based relay selection scheme for a three state Markov channel is presented in Figure 3a,b. Figure 3a represents simulation for BPSK modulation scheme with no source to destination link available while Figure $3 \mathrm{~b}$ represents a simulation for Quadrature Phase Shift Keying (QPSK) modulation.

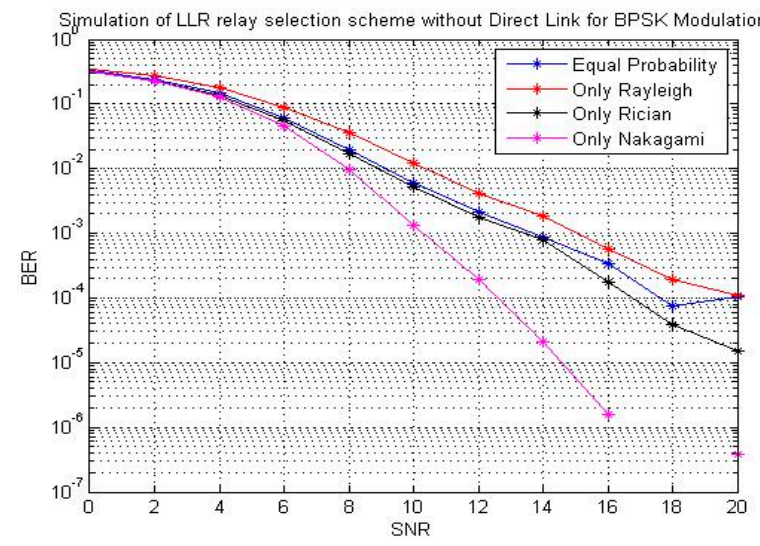

(a)

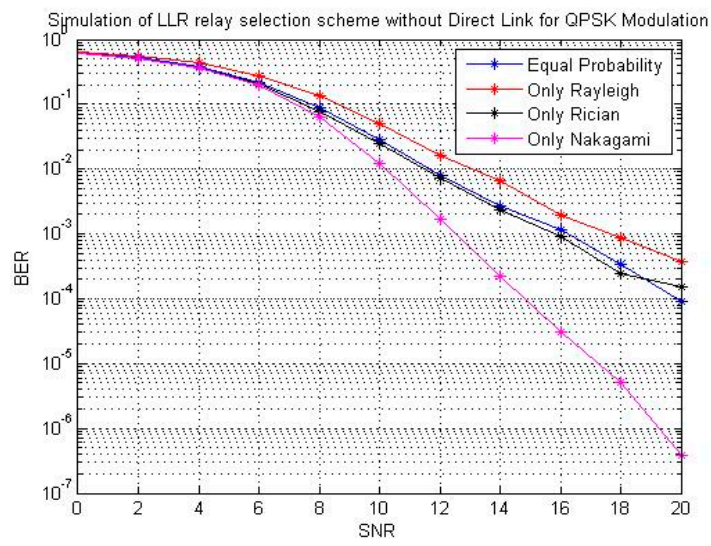

(b)

Figure 3. Bit Error Rate Comparison of a log likelihood ratio (LLR) based relay selection scheme for different values of Transition Probability for (a) Binary Phase Shift Keying (BPSK) Modulation and (b) Quadrature Phase Shift Keying (QPSK) Modulation. Without Source to Destination Link.

In case of BPSK modulation scheme (Figure 3a), the best BER performance is achieved when the transition probability matrix representing Nakagami fading is selected, where BER of the order of $10^{-6}$ is achieved at $16 \mathrm{~dB}$ SNR value. In the case of QPSK modulation it achieves $10^{-6}$ level at $20 \mathrm{~dB}$ SNR for the same parameters.

Figure $4 \mathrm{a}, \mathrm{b}$ presents a BER comparison for a LLR based relay selection scheme with a direct link present between the source to destination node for the BPSK and QPSK modulation scheme, respectively. For this simulation scenario, transition probabilities are set the same as previous the case and LLR is used to find the best relay node. In the case of the BPSK modulation scheme (Figure 4a) the 
best BER performance is achieved when the transition probability matrix representing only Nakagami fading is selected, where BER of the order of approx. $3 \times 10^{-5}$ is achieved at $10 \mathrm{~dB}$ SNR value. While in the case of QPSK modulation, the best achieved BER is approximately $4 \times 10^{-5}$ and is achieved at the $12 \mathrm{~dB}$ SNR value.

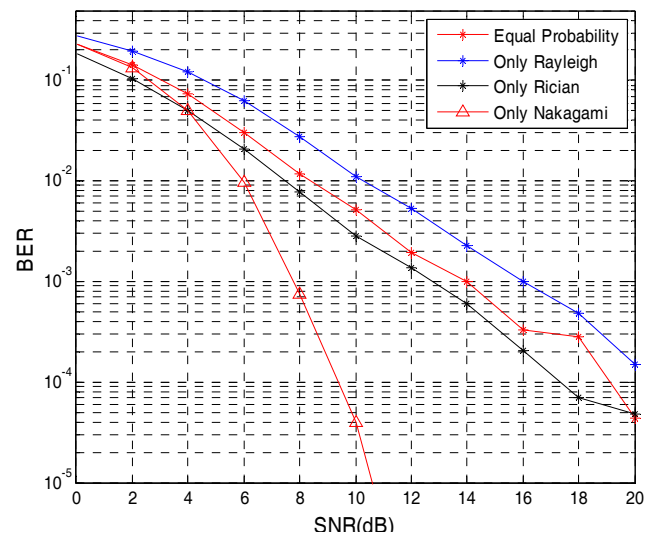

(a)

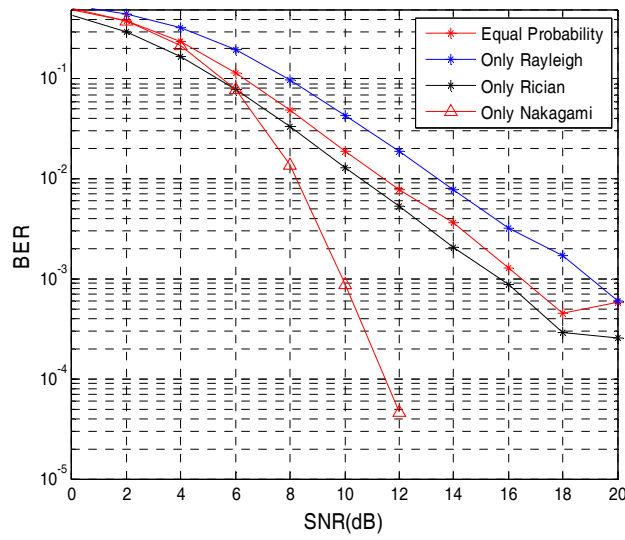

(b)

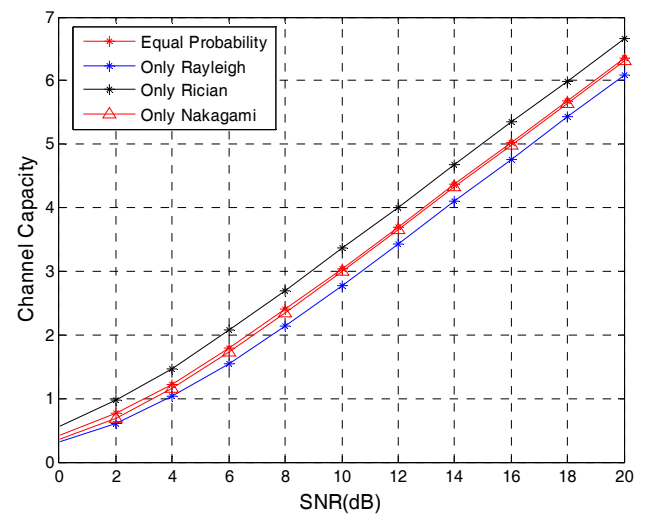

(c)

Figure 4. Comparison of LLR based relay selection scheme for different values of Transition Probability with Source to Destination Link.

Figure 4c presents a channel capacity comparison for LLR based relay selection scheme under different transition probabilities. It can be observed that the maximum achievable channel capacity is provided under only the Rician fading environment, which is found to be approx. $6.5 \mathrm{Bits} / \mathrm{s} / \mathrm{Hz}$ at $20 \mathrm{~dB}$ SNR.

Figure 5 presents (a) LLR based single relay selection scheme (LLR-1 Relay) with source to destination link and (b) LLR based on two relay selection schemes (LLR-2 Relay), with no source to destination link for transition probability and only Nakagami fading environment. The BER comparison of the LLR based relay selection scheme with a source to destination link and without a source to destination link is presented in Figure 5a. An outage comparison for the given scenario is presented in Figure 5b. The threshold of the outage analysis is set to be at an error value greater than $10^{-4}$. It can be seen that the addition of a source to destination link improves the BER of a proposed scheme as a zero-error point with direct link is found to be at $10 \mathrm{~dB}$ SNR, $6 \mathrm{~dB}$ better as compared to a system without a direct link, which is found at $16 \mathrm{~dB}$ SNR for the considered case with transition matrix presenting equal probability for all considered fading scenarios. Clearly the system with a direct link provides better outage behavior when compared to a system without direct link.

Finally, the channel capacity comparison for the considered scenario is presented in Figure 5c. The channel capacity performance of system with direct link is comparably better too. 
Table 2 presents a BER comparison for a LLR based relay selection considering a source to destination link and relay to destination link with transition probability of a Nakagami fading environment (Figure 4a) and LLR based on two relay selection schemes (LLR-2 Relay), with no source to destination link for transition probability with only a Nakagami fading environment (Figure 5a).

Table 2. Bit Error Rate (BER) comparison for scenarios presented in Figures $4 \mathrm{a}$ and $5 \mathrm{a}$.

\begin{tabular}{ccc}
\hline SNR & Figure $5 \mathbf{a}$ & Figure $4 \mathbf{a}$ \\
\hline 0 & 0.231033203125000 & 0.231768750000000 \\
2 & 0.132341406250000 & 0.133731640625000 \\
4 & 0.050177343750000 & 0.050139453125000 \\
6 & 0.009687500000000 & 0.009872265625000 \\
8 & 0.000767578125000 & 0.000750000000000 \\
10 & 0.000024609375000 & 0.000039843750000 \\
12 & 0 & 0.000000390625000 \\
14 & 0 & 0 \\
16 & 0 & 0 \\
18 & 0 & 0 \\
20 & 0 & 0 \\
\hline
\end{tabular}

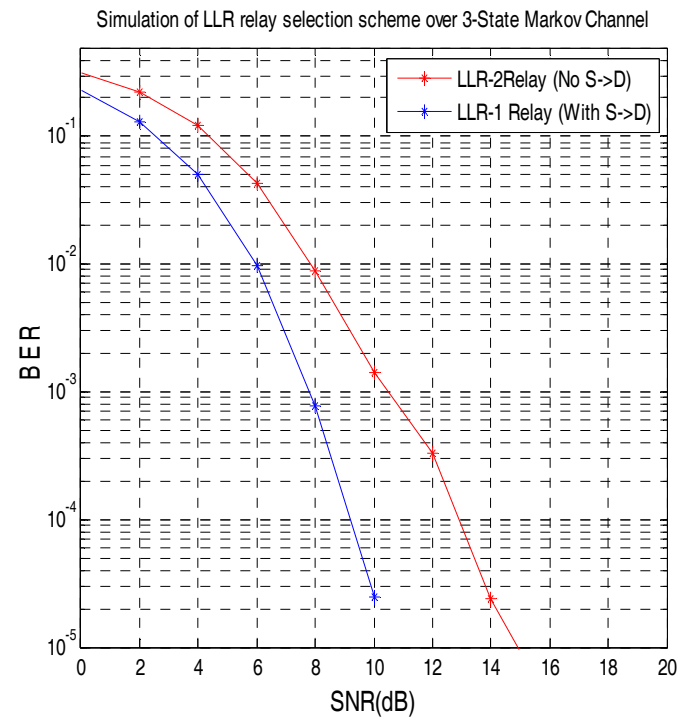

(a)

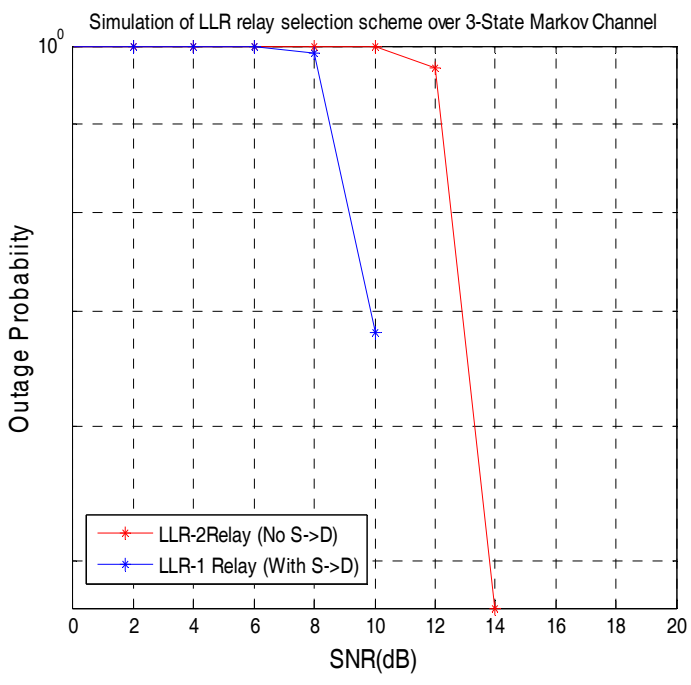

(b)

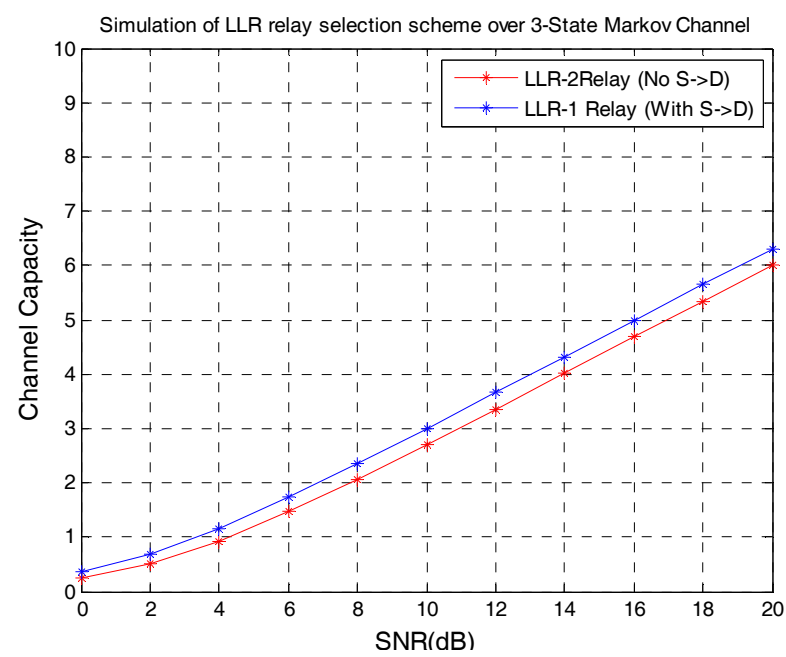

(c)

Figure 5. Comparison of LLR based relay selection scheme with Direct and Indirect Links. 


\section{Conclusions}

This paper proposes an optimal relay selection method among a set of relay nodes using a LLR test. To consider the impact of a common communication scenario, a three state Markov channel based simulation framework is presented to toggle the communication channel among Rayleigh, Rician, and Nakagami-m fading environment. Simulation results on the basis of BER, channel capacity, and outage probability are presented and compared on the basis of the availability of a communication link between the source and destination nodes for a single and multiple relay selection scheme. On the basis of the presented results it is concluded that the proposed scheme provides zero error performance with SNR as low as a $10 \mathrm{~dB}$ fading environment for the case of a single relay with source-destination link case for equal probability for Rayleigh, Rician, and Nakagami. While the maximum instantaneous channel capacity offered by the scheme is approximately $6.5 \mathrm{bits} / \mathrm{s} / \mathrm{Hz}$, which is obtained for $20 \mathrm{~dB}$ SNR. Furthermore, simulation results validate that LLR based relay selection scheme provides robust performance against time-varying channel conditions modelled by a finite state Markov channel.

Author Contributions: Conceptualization, M.S.; Formal analysis, M.S., A.J. and R.G.; Investigation, A.J. and R.G.; Methodology, M.S., A.J. and R.G.; Software, M.S. R.G.; Supervision, A.J. and R.G.; Validation, M.S., A.J. and R.G.; Writing—original draft, M.S.; Writing—review \& editing, A.J. and R.G.

Conflicts of Interest: The authors declare no conflict of interest.

\section{Abbreviations}

The following abbreviations are used in this manuscript:

Symbol Description

$S R_{N} \quad$ received signal at $N^{\text {th }}$ relay in first phase

$\sqrt{s} \quad$ average energy per symbol

$S R_{N} \quad$ channel coefficient present between source and $N^{\text {th }}$ relay

$N_{0} \quad$ Noise variance

$b_{i} \quad$ best relay pair

$L L R_{N} \quad$ Log Likelihood ratio magnitude of signal received at $N^{\text {th }}$ relay in first phase

$\eta_{S R N} \quad$ transmitted symbol from source

$P_{b i} \quad$ bit error probability

$\beta_{i} \quad$ amplification factor

$Z_{R_{i} D} \quad$ received signal at destination after second phase

$\delta_{1}$ and $\delta_{2} \quad$ link weights for MRC

$\gamma_{t} \quad$ Instantaneous SNR

$p_{\gamma_{t}} \quad$ probability density function (PDF) for fading channel

$\mathrm{K} \quad$ ratio between the power in direct path and indirect path in Rican fading

$\Omega \quad$ total available power in Rician Fading

$I_{0}($.$) \quad Zero order Bessal function$

$\mathrm{M} \quad$ shaping parameter in Nakagami fading

$\omega \quad$ controlling spread in Nakagami Fading

$P \quad$ transition probability

$\Pi \quad$ steady state vector

\section{References}

1. Van der Meulen, E. A survey of multi-way channels in information theory: 1961-1976. IEEE Trans. Inf. Theory 1977, 23, 1-37. [CrossRef]

2. Gamal, E.L.; van der Meulen, E. A proof of Marton's coding theorem for the discrete memoryless broadcast channel. IEEE Trans. Inf. Theory 1981, 27, 120-122. [CrossRef]

3. Sendonaris, A.; Erkip, E.; Aazhang, B. User cooperation diversity-Part I: System description. IEEE Trans. Commun. 2003, 51, 1927-1938. [CrossRef] 
4. Sendonaris, A.; Erkip, E.; Aazhang, B. User cooperation diversity-Part II: Implementation aspects and performance analysis. IEEE Trans. Commun. 2003, 51, 1939-1948. [CrossRef]

5. Laneman, J.N.; Wornell, G.W. Exploiting distributed spatial diversity in wireless networks. In Proceedings of the Allerton Conference on Communications, Control and Computing, Monticello, IL, USA, 4-6 October 2000.

6. Laneman, J.N.; Tse, D.N.C.; Wornell, G.W. Cooperative diversity in wireless networks: Efficient protocols and outage behavior. IEEE Trans. Inf. Theory 2004, 50, 3062-3080. [CrossRef]

7. Bletsas, A.; Khisti, A.; Reed, D.P.; Lippman, A. A simple cooperative diversity method based on network path selection. IEEE J. Sel. Areas Commun. 2006, 24, 659-672. [CrossRef]

8. Bletsas, A.; Reed, D.P.; Lippman, A. A simple distributed method for relay selection in cooperative diversity wireless networks, based on reciprocity and channel measurements. In Proceedings of the IEEE 61st Vehicular Technology Conference, Stockholm, Sweden, 30 May 2005; Volume 3, pp. 1484-1488. [CrossRef]

9. Siriwongpairat, W.P.; Himsoon, T.; Su, W.; Liu, K.R. Optimal threshold selection relaying for decode-and-forward cooperation protocol. In Proceedings of the 2006 IEEE Wireless Communications and Networking Conference (WCNC 2006), Las Vegas, NV, USA, 3-6 April 2006; Volume 2, pp. 1015-1020. [CrossRef]

10. Michalopoulos, D.S.; Karagiannidis, G.K.; Tsiftsis, T.A.; Mallik, R.K. Wlc41-1: An optimized user selection method for cooperative diversity systems. In Proceedings of the IEEE Globecom, San Francisco, CA, USA, 27 November-1 December 2006; pp. 1-6. [CrossRef]

11. Brijesh, K.; Kshetrimayum, R.S. Performance Analysis of MIMO Systems with Antenna Selection over Generalized $\kappa-\mu$ Fading Channels. IETE J. Res. 2016, 62, 45-54. [CrossRef]

12. Papadogiannis, A.; Alexandropoulos, G.C. System level performance evaluation of dynamic relays in cellular networks over Nakagami-m fading channels. In Proceedings of the 2009 IEEE 20th International Symposium on Personal, Indoor and Mobile Radio Communications, Tokyo, Japan, 13-16 September 2009; pp. 727-731. [CrossRef]

13. Alexandropoulos, G.C.; Papadogiannis, A.; Sofotasios, P.C. A Comparative Study of Relaying Schemes with Decode and Forward over Nakagami-m Fading Channels. J. Comput. Netw. Commun. 2011, 2011, 560528. [CrossRef]

14. Xu, L.; Zhang, H.; Gulliver, T.A. OP Performance and Power Allocation for DF Relaying M2M Cooperative System. IETE J. Res. 2016, 62, 627-633. [CrossRef]

15. Bai, Z.; Su, Y.; Zhang, N.; Zhang, Q. User-relay Assignment based Antenna Selection Scheme in Multi-user Multi-relay AF Cooperative Communication Network. In Mobile Networks and Applications; Springer: Berlin, Germany, 2017; pp. 1-9.

16. Babak, N.; Han Vinck, A.J. Relay selection in cooperative power line communication: A multi-armed bandit approach. J. Commun. Netw. 2017, 19, 1-9. [CrossRef]

17. Nguyen, M.N.; Nguyen, N.P.; da Costa, D.B.; Nguyen, H.-K.; de Sousa, R.T., Jr. Secure Cooperative Half-Duplex Cognitive Radio Networks with K-th Best Relay Selection. IEEE Access 2017, 5, 6678-6687. [CrossRef]

18. Alexandropoulos, G.C.; Papadogiannis, A.; Berberidis, K. Performance analysis of cooperative networks with relay selection over Nakagami-m fading channels. IEEE Signal Process. Lett. 2010, 17, 441-444. [CrossRef]

19. Alexandropoulos, G.C.; Sofotasios, P.C.; Ho-Van, K.; Freear, S. Symbol Error Probability of DF Relay Selection over Arbitrary Nakagami-Fading Channels. J. Eng. 2013, 2013, 325045. [CrossRef]

20. Varma, U.S.H.; Nikhil, M.V.S.; Manikanta, G.; Kirthiga, S. Cooperative MIMO with relay selection for LTE advanced system. In Proceedings of the 2017 International Conference on Circuit, Power and Computing Technologies (ICCPCT), Kollam, India, 20-21 April 2017.

21. Swain, C.; Kumar, M.; Das, S. Study and Impact of Relay Selection Schemes on Performance of an IEEE 802.16 j Mobile Multihop Relay (MMR) WiMAX Network. In Progress in Intelligent Computing Techniques: Theory, Practice, and Applications; Springer: Singapore, 2018; pp. 491-499.

22. Kim, S.W.; Kim, E.Y. Optimal receive antenna selection minimizing error probability. In Proceedings of the Wireless Communications and Networking IEEE, New Orleans, LA, USA, 16-20 March 2003; Volume 1, pp. 441-447. [CrossRef] 
23. Palat, R.C.; Annamalai, A.; Reed, J.H. Log-likelihood-ratio based selective decode and forward cooperative communication. In Proceedings of the VTC Spring 2008: IEEE Vehicular Technology Conference, Singapore, 11-14 May 2008; pp. 615-618. [CrossRef]

24. Alexan, W.; Mahdy, E.L. A Relay selection based on the log likelihood ratio for cooperative communication networks. In Proceedings of the Signal Processing: Algorithms, Architectures, Arrangements, and Applications (SPA), Poznan, Poland, 22-24 September 2014; pp. 149-153.

25. Wang, H.S.; Moayeri, N. Finite-state Markov channel-a useful model for radio communication channels. IEEE Trans. Vehicular Technol. 1995, 44, 163-171. [CrossRef]

26. Zhang, Q.; Kassam, S.A. Finite-state Markov model for Rayleigh fading channels. IEEE Trans. Commun. 1999, 47, 1688-1692. [CrossRef]

27. Babich, F.; Kelly, O.E.; Lombardi, G. Generalized Markov modeling for flat fading. IEEE Trans. Commun. 2000, 48, 547-551. [CrossRef]

28. Simon, M.K.; Alouini, M.-S. Digital Communication over Fading Channels; John Wiley \& Sons: Hoboken, NJ, USA, 2000; pp. 438-454. ISBN 0-471-31779-9.

(C) 2018 by the authors. Licensee MDPI, Basel, Switzerland. This article is an open access article distributed under the terms and conditions of the Creative Commons Attribution (CC BY) license (http://creativecommons.org/licenses/by/4.0/). 